\title{
Changes in Radiation Dose with Variations in Human Anatomy: Larger and Smaller Normal-Stature Adults
}

\author{
Patrick M. Marine ${ }^{1}$, Michael G. Stabin ${ }^{2}$, Michael J. Fernald ${ }^{3}$, and Aaron B. Brill ${ }^{2}$

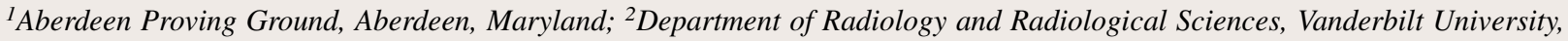 \\ Nashville, Tennessee; and ${ }^{3}$ University of Idaho, Moscow, Idaho
}

\begin{abstract}
A systematic evaluation has been performed to study how specific absorbed fractions (SAFs) vary with changes in adult body size, for persons of different size but normal body stature. Methods: A review of the literature was performed to evaluate how individual organ sizes vary with changes in total body weight of normal-stature individuals. On the basis of this literature review, changes were made to our easily deformable reference adult male and female total-body models. Monte Carlo simulations of radiation transport were performed; SAFs for photons were generated for 10th, 25th, 75th, and 90th percentile adults; and comparisons were made to the reference (50th) percentile SAF values. Results: Differences in SAFs for organs irradiating themselves were between $0.5 \%$ and $1.0 \% / \mathrm{kg}$ difference in body weight, from $15 \%$ to $30 \%$ overall, for organs within the trunk. Differences in SAFs for organs outside the trunk were not greater than the uncertainties in the data and will not be important enough to change calculated doses. For organs irradiating other organs within the trunk, differences were significant, between $0.3 \%$ and $1.1 \% / \mathrm{kg}$, or about $8 \%-33 \%$ overall. Conclusion: The differences are interesting and can be used to estimate how different patients' dosimetry might vary from values reported in standard dose tables.
\end{abstract}

Key Words: radiobiology/dosimetry; radiopharmaceuticals; other; anatomy; radiation dosimetry

J Nucl Med 2010; 51:806-811

DOI: 10.2967/jnumed.109.073007

\section{F} or calculations of radiation dose estimates for radiopharmaceuticals, stylized anatomic models that were developed in the 1960s and 1970s have been used, with tables of specific absorbed fractions (SAFs) (1) and dose factors, perhaps implemented in standardized computer programs such as the MIRDOSE (2) and OLINDA/EXM code (3). The mathematic descriptions of the body and its organs were formulated on the basis of descriptive and schematic materials from general anatomy references. The goal was to

Received Nov. 19, 2009; revision accepted Jan. 26, 2010.

For correspondence or reprints contact: Michael G. Stabin, Department of Radiology and Radiological Sciences, Vanderbilt University, 116121 st Ave. S., Nashville, TN 37232-2675.

E-mail: michael.g.stabin@vanderbilt.edu

COPYRIGHT ( 2010 by the Society of Nuclear Medicine, Inc. make the mathematic equations simple, thus minimizing computing time. Later improvements led to a family of stylized models, which include individuals of both sexes at several ages (4) and pregnant women (5). For several decades, these simplified models have been used for practical applications with the standard mathematic representations of the reference man (6) and other representative phantoms in radiation protection, nuclear medicine, and medical imaging $(7,8)$. However, this stylized modeling approach has obvious shortcomings. Recently, more realistic models, based on imaging data from human subjects, have replaced the traditional, stylized models (9). The newer models include updated anatomic information from reference data on adults and children (10). These models use nonuniform rational B-splines (NURBS) to define body and organ surfaces, as developed by Dr. Paul Segars of Duke University (11). Figure 1 compares the traditional stylized models with the newer realistic models.

The phantoms used in standardized dose assessment are based on a median (i.e., 50th percentile) individual of a large population, for example, adult men or women or children of a particular age. We have now customized a series of phantoms to represent larger and smaller normal-stature individuals. In a separate investigation, we evaluated the influence of obesity on SAFs and dose factors in adults (results to be published separately). Here we describe phantoms that model different body types in a series of percentile height phantoms (10th, 25th, 75th, and 90th percentile adult men and women) based on a normal body mass index (BMI), to evaluate how SAFs may vary with height and weight differences across the human population. The dose to median individuals will continue to be the most widely used method in standardized dosimetry analyses, but an investigation of the variability in these dose values across typical nuclear medicine (and other) populations is important to an understanding of the uncertainty that may exist in these reported values (12).

\section{MATERIALS AND METHODS}

To create this percentile phantom series, we evaluated several literature sources. Dekaban (13) reported on variations in brain 

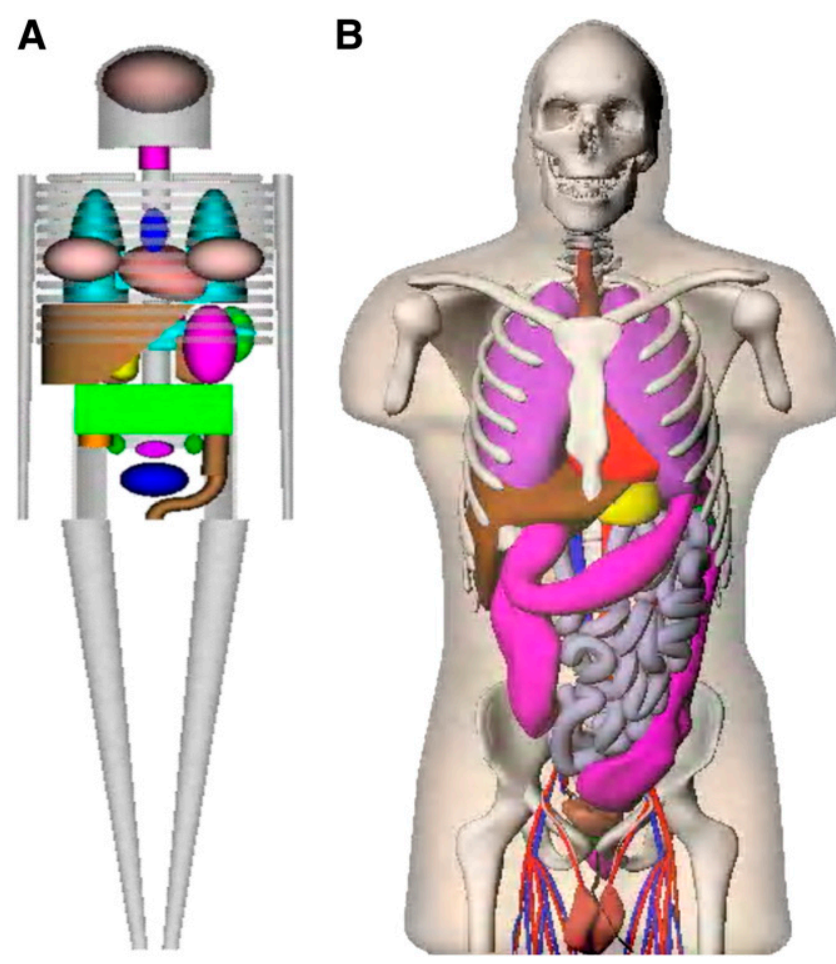

FIGURE 1. Comparison of traditional stylized (A) and realistic human body $(B)$ models used in dose assessment.

weights with human body stature and body weight. Grandmaison et al. (14) provided information on variations in the mass of other body organs in relation to stature and BMI. Anthropometric data from the U.S. Department of Defense (DOD) (15) and Centers for
Disease Control $(16,17)$ were also consulted to evaluate trends in the external measurements of large numbers of adults.

Initially, 50th percentile phantoms were constructed, reflecting the recommended values for organ and body masses (9) of the International Commission on Radiological Protection (ICRP) (10). The male and female adult NURBS phantoms were scaled to represent 25 th and 75 th percentile height phantoms of normal BMI using the anatomic data discussed earlier. The 3 height groupings of Grandmaison et al. (14) for both men and women approximated the 25th, 50th, and 75th percentile heights listed in the Centers for Disease Control and DOD data. Thus, the organ data were correlated to the height percentiles. Several assumptions were made at this point. Because the autopsy data were from nondiseased individuals, we assumed that the percentage difference in organ masses between the middle-height class of autopsy data and the lower and upper classes of autopsy data would be a true approximation of organ masses for the 25th and 75th percentile phantoms. A scaling factor was determined for each organ from this relative percentage change. For organs not represented in the autopsy data, such as intestines, the volume was approximated by a $5 \%$ increase or decrease from the reference values. The eyes and other head organs were scaled $\pm 3 \%$ to stay consistent with the brain scaling.

Differences in the skeletal system relative to height were scaled to external reference markers. For example, the chest cavity and rib cage were scaled 3-dimensionally to the percentage difference in chest circumference. Once the 25th and 75th percentile phantoms were completed, additional 10th and 90th percentile phantoms were developed. Fewer autopsy data were available for these models, so a different methodology was used. The heights for the 10th and 90th percentile phantoms were taken from the DOD-HDBK-743A (15), and BMIs of 24 for the men and 23 for the women were assumed. The total mass of the individual phantom was then calculated. Scaling factors were then applied

\section{TABLE 1. Selected Organ Masses (g) for Adult Male Models}

\begin{tabular}{|c|c|c|c|c|c|}
\hline Organ & 10th percentile & 25th percentile & 50th ICRP reference & 75th percentile & 90th percentile \\
\hline Adrenals & 11.88 & 12.65 & 14 & 14.93 & 15.94 \\
\hline Brain & 1,291 & 1,405 & 1,450 & 1,493 & 1,527 \\
\hline Esophagus & 36.17 & 39.29 & 40 & 43.26 & 46.52 \\
\hline Eyes & 13.6 & 14.82 & 15 & 15.16 & 16.82 \\
\hline Gallbladder & 62.54 & 68.13 & 68 & 71.65 & 79.82 \\
\hline Stomach & 341 & 370.8 & 400 & 419.1 & 443.7 \\
\hline Small intestine & 595 & 648.7 & 650 & 682.3 & 779.4 \\
\hline Right colon & 141 & 154 & 150 & 170 & 186 \\
\hline Left colon & 133 & 146 & 150 & 159 & 174 \\
\hline Rectosigmoid & 61.5 & 66.34 & 70 & 75.13 & 81.78 \\
\hline Heart & 740 & 804.5 & 840 & 833 & 978.3 \\
\hline Kidneys & 278.4 & 302.7 & 310 & 322.5 & 366.4 \\
\hline Liver & 1,489 & 1,621 & 1,800 & 1,977 & 2,206 \\
\hline Lungs & 1,068 & 1,162 & 1,200 & 1,344 & 1,502 \\
\hline Pancreas & 127.5 & 138.2 & 140 & 144 & 161 \\
\hline Prostate & 14.94 & 16.32 & 17 & 18 & 19.49 \\
\hline Salivary glands & 76.76 & 82.63 & 85 & 88.11 & 92.22 \\
\hline Skeleton & 8,289 & 8,990 & 10,500 & 10,781 & 11,800 \\
\hline Spleen & 112.3 & 121.5 & 150 & 172 & 196.1 \\
\hline Testes & 30.56 & 33.16 & 35 & 37.28 & 39.73 \\
\hline Thymus & 21.68 & 23.3 & 25 & 26.53 & 27.76 \\
\hline Thyroid & 18.42 & 19.99 & 20 & 20.54 & 21.04 \\
\hline Urinary bladder & 45.05 & 48.63 & 50 & 53.72 & 59.02 \\
\hline Total body & 63,560 & 69,170 & 73,000 & 76,790 & 91,720 \\
\hline
\end{tabular}




\section{TABLE 2. Selected Organ Masses (g) for Adult Female Models}

\begin{tabular}{|c|c|c|c|c|c|}
\hline Organ & 10th percentile & 25th percentile & 50th percentile & 75th percentile & 90th percentile \\
\hline Adrenals & 11.57 & 12.51 & 13 & 13.7 & 15.33 \\
\hline Brain & 1,150 & 1,243 & 1,300 & 1,317 & 1,361 \\
\hline Esophagus & 32.41 & 34.7 & 35 & 37.75 & 40.78 \\
\hline Eyes & 14.11 & 15.37 & 15 & 15.76 & 16.7 \\
\hline Gallbladder & 50.32 & 54.63 & 56 & 61.66 & 67.73 \\
\hline Stomach & 336.6 & 363.1 & 370 & 400.2 & 438.4 \\
\hline Small intestine & 570.9 & 615.9 & 600 & 671.8 & 737.6 \\
\hline Right colon & 133 & 142 & 145 & 151 & 164 \\
\hline Left colon & 136 & 145 & 145 & 154 & 174 \\
\hline Rectosigmoid & 63.7 & 69.16 & 70 & 76.25 & 84.52 \\
\hline Heart & 546.9 & 588.8 & 620 & 628.6 & 686.4 \\
\hline Kidneys & 229.1 & 246.7 & 276 & 306.8 & 334.7 \\
\hline Liver & 1,150 & 1,238 & 1,400 & 1,556 & 1,703 \\
\hline Lungs & 816.2 & 880 & 950 & 1,011 & 1,106 \\
\hline Ovaries & 7.74 & 8.12 & 11 & 11.45 & 12.63 \\
\hline Pancreas & 103.3 & 110.6 & 120 & 138.6 & 148.4 \\
\hline Salivary glands & 65.27 & 69.9 & 70 & 74.9 & 78.24 \\
\hline Skeleton & 5,849 & 6,284 & 7,800 & 7,470 & 8,240 \\
\hline Spleen & 108.2 & 116.4 & 130 & 152.5 & 165.6 \\
\hline Thymus & 17.37 & 18.74 & 20 & 20.83 & 22.56 \\
\hline Thyroid & 16.27 & 17.49 & 17 & 17.59 & 18.21 \\
\hline Urinary bladder & 36.03 & 39.5 & 40 & 44.5 & 48.03 \\
\hline Uterus & 70.05 & 75.3 & 80 & 88.03 & 95.35 \\
\hline Total body & 50,680 & 54,620 & 60,000 & 65,340 & 71,420 \\
\hline
\end{tabular}

to increase or decrease each organ and bone so they would sum to the indicated mass of the 10th or 90th percentile phantom of the chosen BMI. Additionally, the thicknesses of the weight-bearing bones for the 90th percentile phantom were increased to compensate for the additional frame mass.

Once the phantoms had been scaled using the NURBS scaling tool, the file was voxelized and introduced into the Geant 4

\section{TABLE 3. Organ Density Values Used in \\ Anthropomorphic Models}

\begin{tabular}{|c|c|}
\hline Organ & Density $\left(\mathrm{g} \cdot \mathrm{cm}^{-3}\right)$ \\
\hline Adrenals & 1.02 \\
\hline Salivary glands & 1.045 \\
\hline Esophagus & 1.045 \\
\hline Stomach & 1.045 \\
\hline Small intestine & 1.045 \\
\hline Large intestine & 1.045 \\
\hline Rectosigmoid & 1.045 \\
\hline Liver & 1.045 \\
\hline Gallbbladder & 1.045 \\
\hline Pancreas & 1.045 \\
\hline Brain & 1.04 \\
\hline Heart & 1.03 \\
\hline Eyes & 1.026 \\
\hline Lungs & 0.30 \\
\hline Skeleton & 1.3 \\
\hline Spleen & 1.06 \\
\hline Thymus & 1.025 \\
\hline Thyroid & 1.05 \\
\hline Kidneys & 1.05 \\
\hline Bladder & 1.03 \\
\hline Testes & 1.04 \\
\hline Prostate & 1.03 \\
\hline Ovaries & 1.05 \\
\hline Uterus & 1.05 \\
\hline
\end{tabular}

radiation transport code (18). SAFs were generated for most body organs, and comparisons were made to SAFs from both the Cristy-Eckerman phantoms and the 50th percentile NURB ICRP 89 phantoms. Additionally, the SAFs for the 10th, 25th, 75th, and 90th percentile phantoms were plotted together, and the trends were analyzed. Direct comparisons were made with SAF values of the 50th percentile ICRP 89 models (9). The percentage differences in SAF values across the percentiles were estimated for several organ pairs as arithmetic averages across all energies and expressed as absolute percentage differences and percentage differences per kilogram difference in phantom total body mass.

\section{RESULTS}

Tables 1 and 2 compare organ masses in the 10th, 25th, 50th, 75th, and 90th percentile adult men and women,

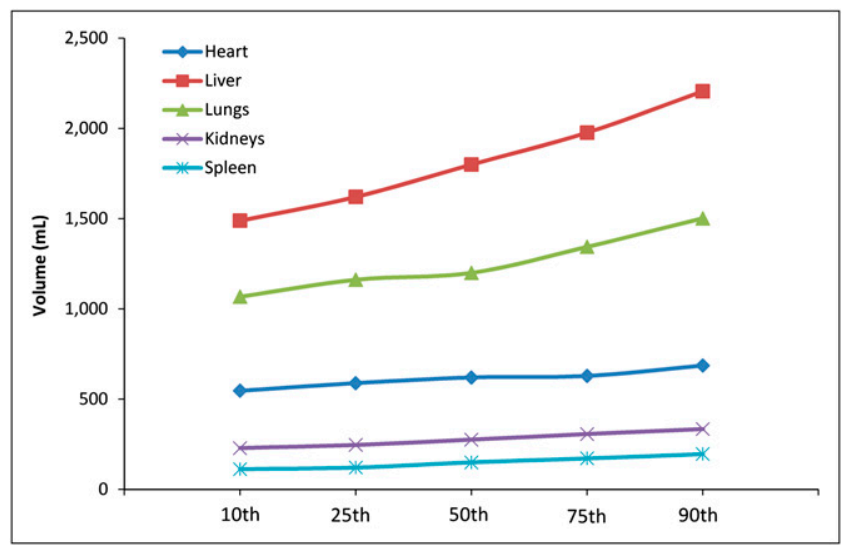

FIGURE 2. Selected organ masses, adult male models. 


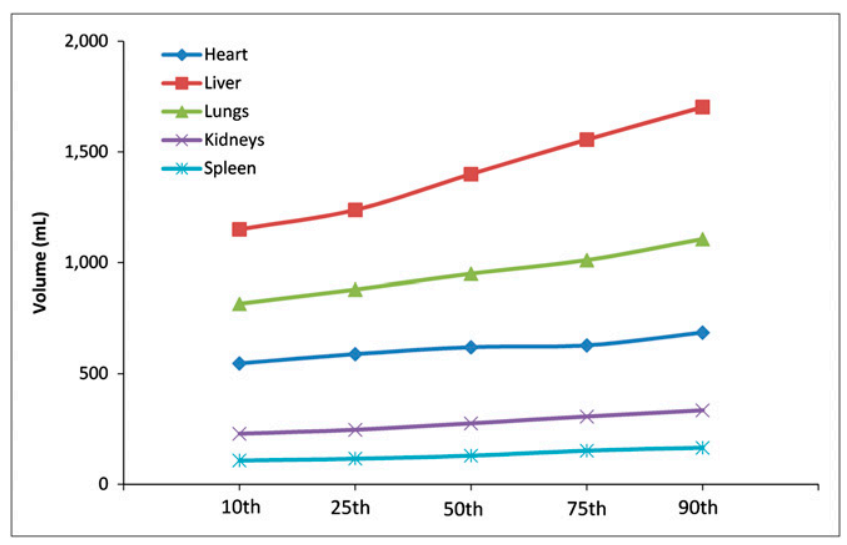

FIGURE 3. Selected organ masses, adult female models.

respectively. Table 3 shows tissue densities assumed in the simulations (10). Figures 2 and 3 show the difference in mass for 5 organs in the adult men and women, respectively, across percentile groups. Figures 4 and 5 show selected SAF plots for the male and female models, respectively, across percentiles. Tables 4-7 show the percentage differences - both absolute and body weightbased-for selected organ pairs from the 10th to the 90th percentile male and female phantoms.

\section{DISCUSSION}

The literature surveyed suggested that certain organs vary much more than others with changes in body size.
For example, according to the nondiseased autopsy data from Grandmaison et al. (14), the male spleen varied by about $20 \%$ between the 50th percentile height and the 75th percentile height, whereas the heart varied by only $5.8 \%$. This difference may be due to functional reserve capacities, metabolic demands, or other variables. However, the autopsy sample set was relatively small (684 cases), so some statistical uncertainty is clearly associated with the data. In addition, the assignment of the 75th and 25th percentiles to the autopsy data was an approximation; with a larger set of raw nondiseased data, the assigned percentiles could be refined. Grandmaison et al. (14) also noted that although most organs correlate more strongly with height, some correlate more strongly with BMI, as the heart does. Because this effort was focused on normal-BMI individuals of differing heights, it was expected that given a constant BMI some organs would vary more than others with changes in height.

The SAFs generally decrease with increases in percentile size, because the organ masses are larger, and this difference is more important than the small reductions in SAFs that occur because of slightly greater organ separation. Differences in SAFs for an organ irradiating itself were between $0.5 \%$ and $1.0 \% / \mathrm{kg}$ difference in body weight, from $15 \%$ to $30 \%$ overall, in the organs shown in Tables 4-7. Organs outside the main trunk area showed differences that were not perceptible within the uncertainties of the data; these organs may thus be considered less important than organs in the trunk when one is determining differences in calculated doses that result from the use of SAFs. For
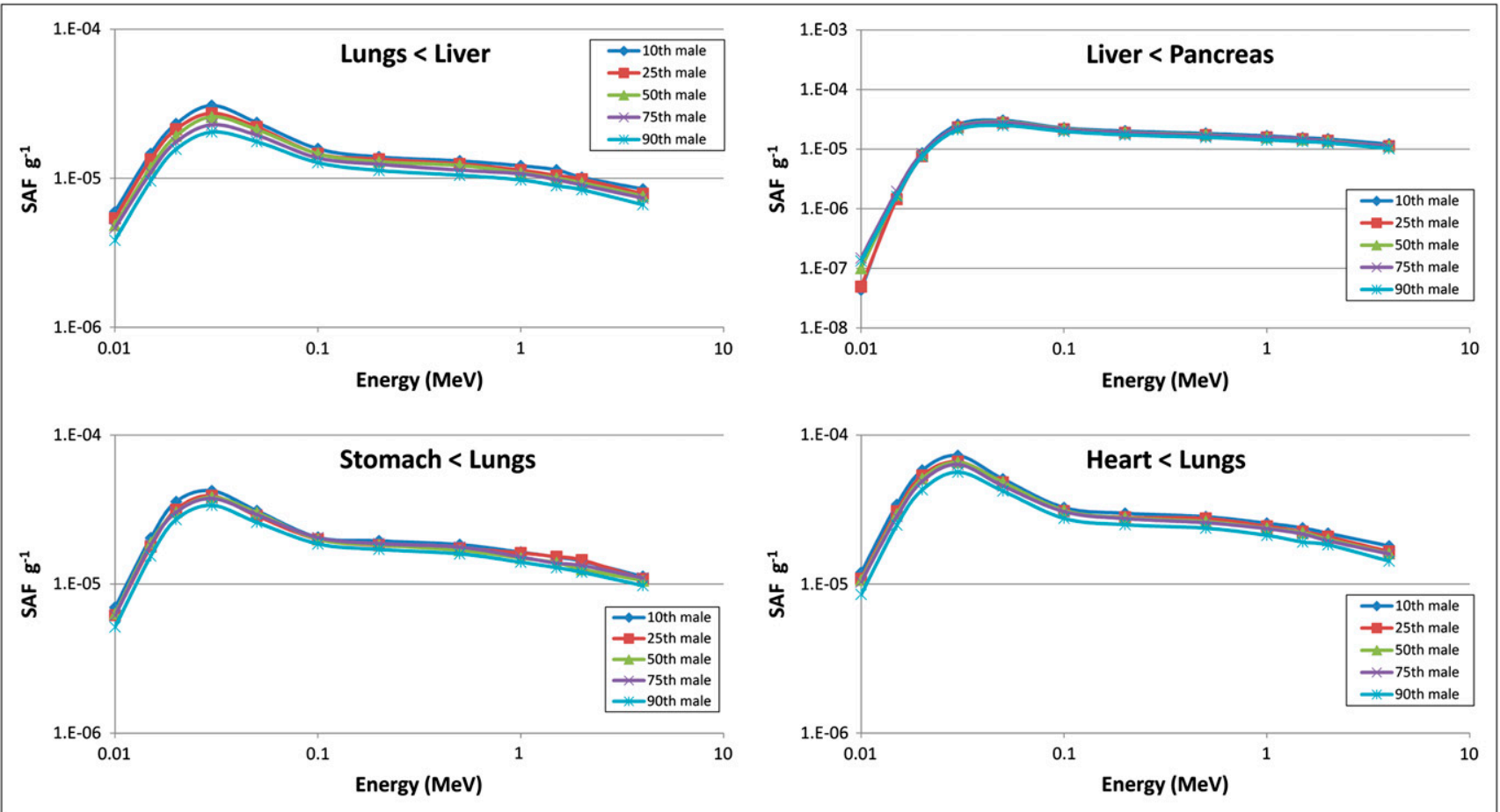

FIGURE 4. Selected SAF plots, adult male. 

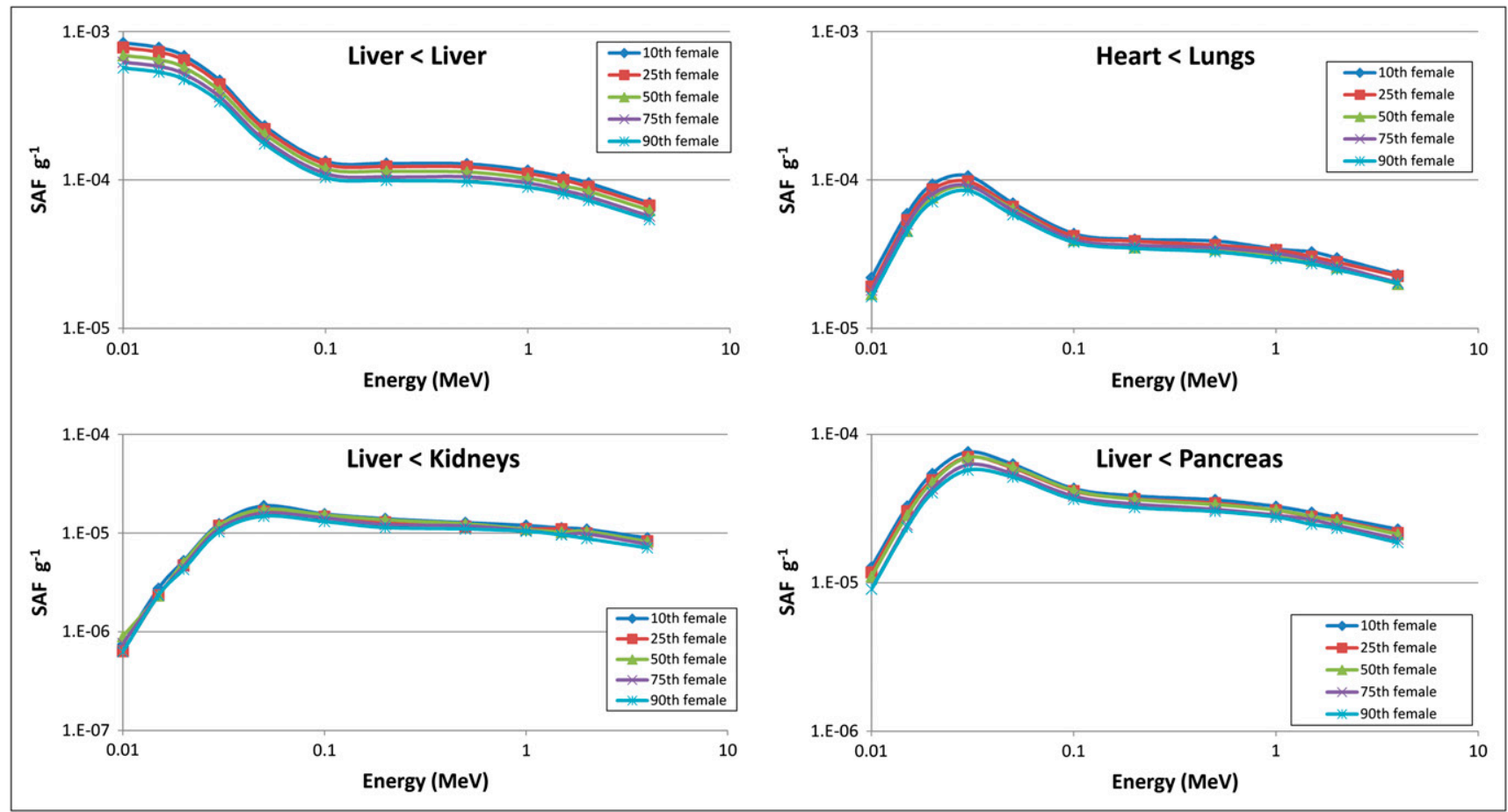

FIGURE 5. Selected SAF plots, adult female.

organs irradiating other organs in the trunk area, differences were between $0.3 \%$ and $1.1 \% / \mathrm{kg}$, or about $8 \%-33 \%$ overall. Given the uncertainties inherent in the analysis, numbers for all cases are roughly similar, and no significant differences were seen between values for the male and female models.

We also calculated for several cases the variation of the SAFs for each phantom from the 50th percentile numbers in each direction and also the difference between each set of phantom results, in steps from the 10th to the 90th percentile models (data not shown). The results generally varied in an approximately uniform manner from phantom to phantom, suggesting that the absolute difference per kilogram from the 10th to the 90th percentage models is fairly constant as the phantom size varies. This constancy is seen in the plots of the SAFs for the different phantoms and was the basis for our reporting the average difference per kilogram difference in total body mass.

\begin{tabular}{|c|c|c|c|c|c|c|}
\hline Organ & Kidneys & Liver & Lungs & Pancreas & Heart & Spleen \\
\hline Kidneys & 0.59 & 0.85 & 0.34 & 0.63 & 0.44 & 0.75 \\
\hline Liver & 1.01 & 0.89 & 0.91 & 1.09 & 0.62 & 1.02 \\
\hline Lungs & 0.46 & 0.74 & 0.69 & 0.52 & 0.62 & 0.21 \\
\hline Pancreas & 0.47 & 1.08 & 0.65 & 0.52 & 0.39 & 0.72 \\
\hline Heart & 0.40 & 0.45 & 0.66 & 0.51 & 0.62 & 0.39 \\
\hline Spleen & 0.62 & 0.72 & 0.31 & 0.70 & 0.51 & 1.09 \\
\hline
\end{tabular}

Differences in SAFs with percentile height differences are only one variable contributing to the overall uncertainty in a radiopharmaceutical dose estimate. One of the major uncertainties in the evaluation of radiation doses for radiopharmaceuticals is the biokinetic model used to calculate the dose (12); this analysis suggested that overall uncertainties of up to a factor of 2 or more may be present in reported dose estimates, with a large degree of uncertainty being attributed to variations in individual biokinetics and organ sizes. This present study partially addresses the impact of the latter variable. If careful patient-specific dosimetry is performed, with attention paid to accurate data acquisition, analysis, and measurement of individual organ volumes, many of the biokinetic model uncertainties can be minimized, and the total uncertainty in the individual dose estimate can be reduced to perhaps $\pm 10 \%-20 \%$ (12). Without individualized dosimetry (which is routine in radiation therapy but not in diagnostic applications of radiopharmaceuticals), the variations shown here in SAFs

\begin{tabular}{|c|c|c|c|c|c|c|}
\hline Organ & Kidneys & Liver & Lungs & Pancreas & Heart & Spleen \\
\hline Kidneys & 17.7 & 25.4 & 10.2 & 18.9 & 13.1 & 22.6 \\
\hline Liver & 30.4 & 26.7 & 27.4 & 32.8 & 18.6 & 30.7 \\
\hline Lungs & 13.7 & 22.2 & 20.6 & 15.6 & 18.6 & 6.4 \\
\hline Pancreas & 14.0 & 32.3 & 19.4 & 15.5 & 11.6 & 21.5 \\
\hline Heart & 12.1 & 13.6 & 19.8 & 15.4 & 18.7 & 11.7 \\
\hline Spleen & 18.7 & 21.7 & 9.3 & 21.1 & 15.4 & 32.6 \\
\hline
\end{tabular}


TABLE 6. Percentage Difference in SAFs per Kilogram

Difference in Total Body Mass from 10th to 90th

Percentile Phantoms, Adult Female

Organ Kidneys Liver Lungs Pancreas Heart Spleen

$\begin{array}{lllllll}\text { Kidneys } & 0.77 & 0.58 & 0.37 & 0.57 & 0.47 & 0.50\end{array}$

$\begin{array}{lllllll}\text { Liver } & 0.56 & 0.86 & 0.61 & 0.68 & 0.26 & 0.74\end{array}$

$\begin{array}{lllllll}\text { Lungs } & 0.29 & 0.58 & 0.62 & 0.51 & 0.60 & 0.45\end{array}$

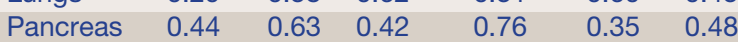

$\begin{array}{lllllll}\text { Heart } & 0.26 & 0.32 & 0.62 & 0.34 & 0.52 & 0.40\end{array}$

$\begin{array}{lllllll}\text { Spleen } & 0.50 & 0.64 & 0.48 & 0.51 & 0.36 & 0.87\end{array}$

contribute at most about $30 \%$ to the overall possible factor of 2 in uncertainties in the dose values. The changes in SAFs found here will thus be of minor importance to the evaluation of the average radiation doses patients are receiving from diagnostic radiopharmaceuticals, for which standardized dose estimates are applied from resources such as the ICRP Task Group Tables (8) and the RADAR Web site (http://www.doseinfo-radar.com) (19).

\section{CONCLUSION}

A systematic evaluation has been performed to study how SAF values vary with changes in adult body size, for persons of different sizes but normal stature. We have studied separately the effects of adult obesity on SAF values, and that study, and a paper that evaluates the actual change in dose estimates for several important pharmaceuticals, will be published separately. Within the trunk, differences in SAFs for organs irradiating themselves were between $0.5 \%$ and $1.0 \% / \mathrm{kg}$ difference in body weight, from $15 \%$ to $30 \%$ overall. Organs outside the trunk showed changes that were not perceptible given the uncertainties of the data and so may be considered to be less important to variations in calculated doses that result from the use of SAFs. For organs irradiating other organs in the trunk, differences were between $0.3 \%$ and $1.1 \% / \mathrm{kg}$, or about $8 \%-33 \%$ overall. The differences are interesting and can help us understand how a patient's dosimetry might vary from values reported in standard dose tables $(8,19)$.

\section{REFERENCES}

1. Snyder WS, Ford MR, Warner GG. Estimates of Specific Absorbed Fractions for Photon Sources Uniformly Distributed in Various Organs of a Heterogeneous Phantom. MIRD pamphlet no. 5, revised. New York, NY: Society of Nuclear Medicine; 1978.

2. Stabin MG. MIRDOSE: the personal computer software for use in internal dose assessment in nuclear medicine. J Nucl Med. 1996;37:538-546.
TABLE 7. Absolute Percentage Difference in SAFs from

10th to 90th Percentile Phantoms, Adult Female

Organ Kidneys Liver Lungs Pancreas Heart Spleen

$\begin{array}{lllllll}\text { Kidneys } & 23.0 & 17.3 & 11.0 & 17.0 & 14.2 & 14.9\end{array}$

$\begin{array}{lllllll}\text { Liver } & 16.8 & 25.8 & 18.3 & 20.5 & 7.9 & 22.1\end{array}$

$\begin{array}{lrrrrrr}\text { Lungs } & 8.8 & 17.4 & 18.7 & 15.2 & 17.9 & 13.4\end{array}$

$\begin{array}{lrrrrrr}\text { Pancreas } & 13.2 & 18.8 & 12.7 & 22.9 & 10.4 & 14.4\end{array}$

$\begin{array}{lllllll}\text { Heart } & 7.8 & 9.7 & 18.5 & 10.2 & 15.5 & 12.1\end{array}$

$\begin{array}{lllllll}\text { Spleen } & 15.1 & 19.2 & 14.3 & 15.2 & 10.7 & 26.0\end{array}$

3. Stabin MG, Sparks RB, Crowe E. OLINDA/EXM: the second-generation personal computer software for internal dose assessment in nuclear medicine. J Nucl Med. 2005;46:1023-1027.

4. Cristy M, Eckerman K. Specific Absorbed Fractions of Energy at Various Ages from Internal Photons Sources. ORNL/TM-8381 V1-V7. Oak Ridge, TN: Oak Ridge National Laboratory; 1987.

5. Stabin M, Watson E, Cristy M, et al. Mathematical Models and Specific Absorbed Fractions of Photon Energy in the Nonpregnant Adult Female and at the End of Each Trimester of Pregnancy. ORNL/TM-12907. Oak Ridge, TN: Oak Ridge National Laboratory; 1995.

6. International Commission on Radiological Protection (ICRP). Report of the Task Group on Reference Man. ICRP publication 23. Oxford, U.K.: Pergamon Press; 1975 .

7. International Commission on Radiation Units and Measurements (ICRU). Measurement of Dose Equivalents from External Photon and Electron Radiations. ICRU report 47. Bethesda, MD: ICRU; 1992.

8. International Commission on Radiological Protection (ICRP). Radiation Dose to Patients from Radiopharmaceuticals: Addendum 2 to ICRP Publication 53, Also Includes Addendum 1 to ICRP Publication 72. ICRP publication 80. Oxford, U.K.: Pergamon Press; 1998.

9. Stabin MG, Emmons MA, Segars WP, Fernald MJ. The Vanderbilt University reference adult and pediatric phantom series. In: Xu XG, ed. Handbook of Anatomical Models for Radiation Dosimetry. Philadelphia, PA: Taylor \& Francis, Inc.; 2009:337-346.

10. International Commission on Radiological Protection (ICRP). Basic Anatomical and Physiological Data for Use in Radiological Protection: Reference Values. ICRP publication 89. Oxford, U.K.: Elsevier Science, Ltd.; 2003.

11. Segars JP. Development and Application of the New Dynamic NURBS-Based Cardiac-Torso (NCAT) Phantom [Ph.D. dissertation]. Chapel Hill, NC: The University of North Carolina; 2001.

12. Stabin MG. Uncertainties in internal dose calculations for radiopharmaceuticals. J Nucl Med. 2008;49:853-860.

13. Dekaban AS. Changes in brain weights during the span of human life: relation of brain weights to body heights and body weights. Ann Neurol. 1978;4:345-356.

14. Grandmaison GL, Clairand I, Durigon M. Organ weight in 684 adult autopsies: new tables for a Caucasoid population. Forensic Sci Int. 2001;119:149-154.

15. U.S. Department of Defense (DOD). Anthropometry of U.S. Military Personnel. DOD-HDBK-743A. Washington, D.C.: DOD; 1991.

16. Ogden CL, Fryar CD, Carroll MD, Flegal KM. Mean Body Weight, Height, and Body Mass Index, United States 1960-2002. Advance Data from Vital and Health Statistics, no. 347. Hyattsville, MD: National Center for Health Statistics; 2004.

17. McDowell MA, Fryar CD, Hirsch R, Ogden CL. Anthropometric Reference Dato for Children and Adults: U.S. Population, 1999-2002. Advance Data from Vital and Health Statistics, no. 361. Hyattsville, MD: National Center for Health Statistics; 2005.

18. Agostinelli $\mathrm{S}$, Allison $\mathrm{J}$, Amako $\mathrm{K}$, et al. $\mathrm{G}_{\mathrm{EANT}}$ 4: a simulation toolkit. Nucl Instrum Meth A. 2003;506:250-303.

19. Stabin MG, Siegel JA. Physical models and dose factors for use in internal dose assessment. Health Phys. 2003;85:294-310. 